\title{
Hydrogen Sensor Using Thin Film with Interspace
}

\author{
Ken Sawaide, Tatsuro Yamada, Kazuhiro Hara \\ Tokyo Denki University, 5 Senju-Asahi-cho, \\ Adachi-ku, Tokyo 120-8551 Japan \\ hara@eee.dendai.ac.jp
}

\begin{abstract}
A Hydrogen sensor using a multiple layered thin film with an interspace is presented. The material for the sensing film is $\mathrm{WO}_{3}$-based. The sensor is highly sensitive to hydrogen gas without using any noble catalysts that are expensive and may lead to degradation of the sensitivity. The porous structure of the sensing film with an interspace in addition to the porous structure of the sensing film itself enhances the effective surface area and thus the sensitivity to hydrogen gas. The selectivity to hydrogen gas is also enhanced by the proposed structure. The response time and recovery time are $15 \mathrm{~s}$ and $20 \mathrm{~s}$, respectively. All films are deposited by r.f. sputtering and an interspace is released by sacrificial layer etching of $\mathrm{a} \mathrm{SiO}_{2}$ film.
\end{abstract}

Key words: hydrogen sensor, thin film, metal oxide, $\mathrm{WO}_{3}$, multiple layer, interspace

\section{Introduction}

A wide variety of hydrogen sensors have been investigated[1]. Among them semiconducting metal-oxide sensors have the advantage of a simple structure, small size and low-cost. Thinfilm sensors are especially advantageous, as they can be fabricated using an all dry process that is compatible with the I.C. fabrication process. However, in general the sensitivity and selectivity of semiconductor thin-film gas sensors are not satisfactory[2]. This paper presents a novel method to improve the sensitivity and selectivity of metal oxide thin-film gas sensors without noble metals that are expensive and tend to cause the sensitivity to gradually deteriorate in the presence of silicon based gases.

\section{Sensor configuration}

A schematic top and cross-sectional view of a proposed sensor(sensor A) is shown in Fig. 1. The sensing film has a two-layered structure with an interspace. The material for the sensing film and the electrode is $\mathrm{WO}_{3}+\mathrm{TiO}_{2}(10 \mathrm{~mol} \%)$ and $\mathrm{Pt}+\mathrm{W}(5 \mathrm{~mol} \%)$, respectively. A schematic cross-sectional view of another sensor(sensor B) that has a four-layered film is shown in Fig. 2 .

The surface morphology of a $\mathrm{WO}_{3}$-based film after annealing at $600^{\circ} \mathrm{C}$ is shown in Fig.3. The sensing film itself is porous.

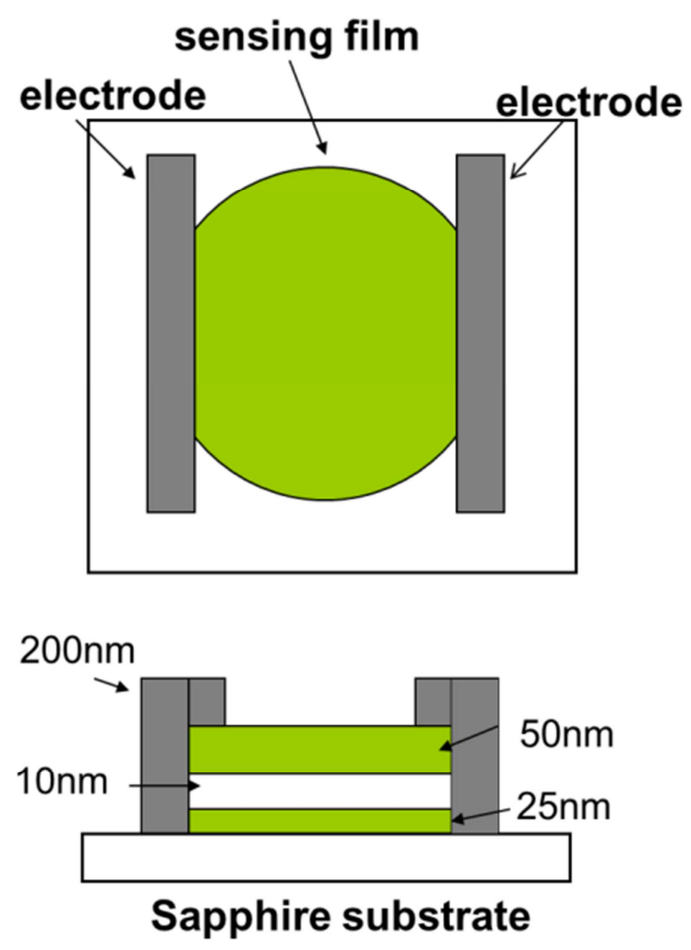

Fig. 1 Schematic top and cross-sectional view of a proposed hydrogen sensor using a doublelayered metal-oxide thin-film and a Ni-deposited porous alumina(sensor $A$ ) 


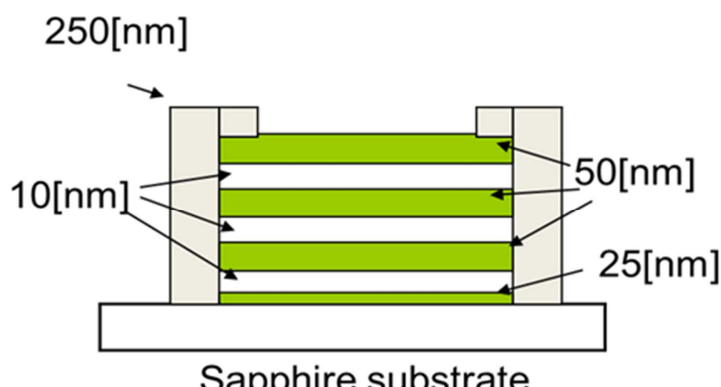

Sapphire substrate

Fig.2 Schematic cross-sectional view of a metal oxide thin-film gas sensor using a four-layered film with an interspace(sensor B).

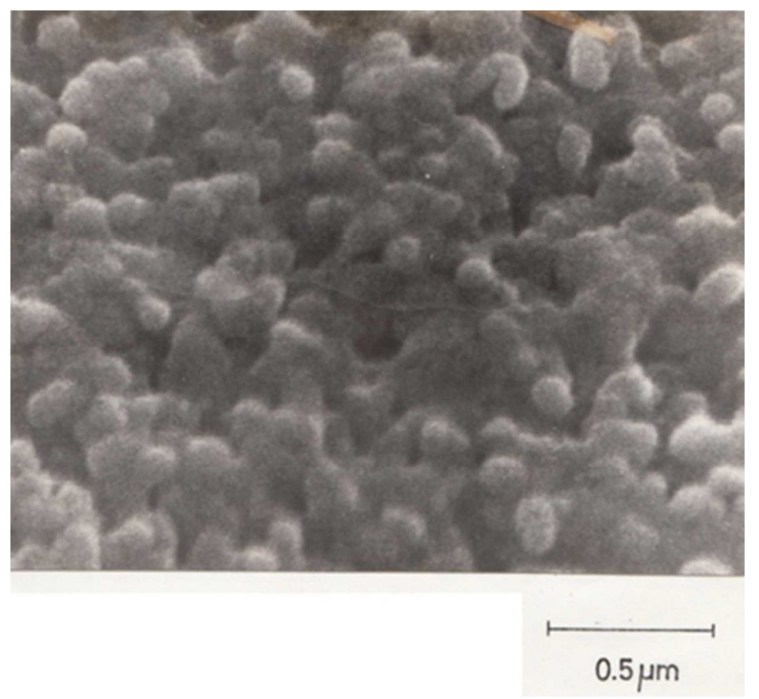

Fig.3 Surface morphology of a $\mathrm{WO}_{3}$-based film after annealing at $600^{\circ} \mathrm{C}$.

\section{Sensor fabrication}

All films in Fig. 1 and 2 were successively deposited by r.f. sputtering on a sapphire substrate $(10 \mathrm{~mm} \times 10 \mathrm{~mm} \times 1 \mathrm{~mm}$, c-plane, mirror polished). The interspace with a thickness of $10 \mathrm{~nm}$ is released by etching the deposited $\mathrm{SiO}_{2}$ film by $1 \%$ of fluoric acid for about 1 minute(sensor $A$ ) and about 3 minutes(sensor B). Finally the sensing film is annealed in air at $600^{\circ} \mathrm{C}$ for $6 \mathrm{~h}$ for the recovery from various defects and stabilization of the crystal structure.

In the etching process, fluoric acid is likely to pass through the pores inside the $\mathrm{WO}_{3}$ film in addition to the interspace at both side edges of the film. A schematic microscopic top and cross-sectional view of a sensing film in sensor A is shown in Fig. 4. Some part of $\mathrm{SiO}_{2}$ film is assumed to remain undissolved, acting as supporting rods of the upper $\mathrm{WO}_{3}$-based film.

A schematic microscopic top and crosssectional view of a sensing film in sensor $B$ is shown in Fig. 5.

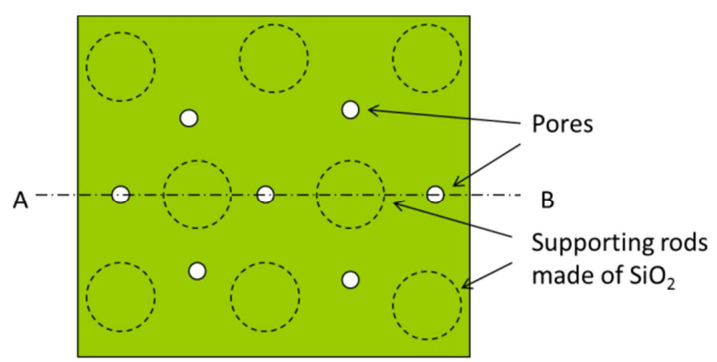

(a) Top view

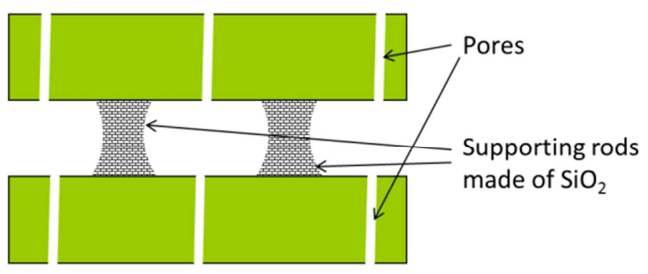

(b) Cross-sectional view along A-B

Fig.4 Schematic microscopic top and crosssectional view of a sensing film (sensor $A$ ).

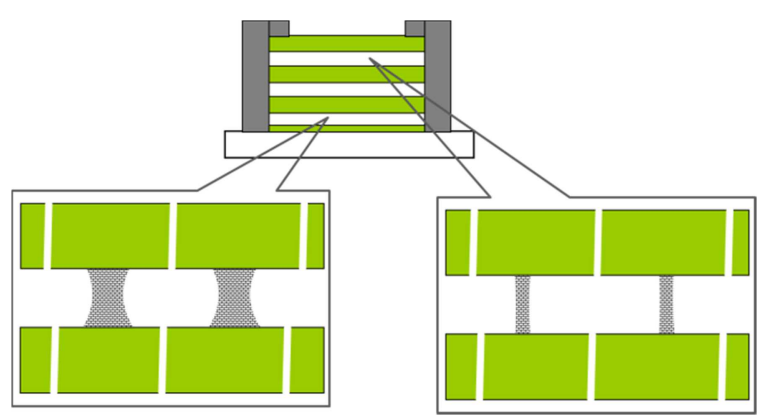

Fig.5 Schematic microscopic cross-sectional view of a sensing film (sensor $B$ ).

Since fluoric acid penetrates through the top film, the upper supporting rods are thin or broken away because of prolonged etching. Thus the sensor is fragile.

\section{Experimental results}

The temperature dependence of the sensitivity to hydrogen is shown in Fig. 6 for four kinds of sensors. Here the sensitivity is defined as the ratio of the resistance in air to that in gas. The sensitivity to hydrogen increased after sacrificial layer etching to create an interspace and was higher than that of a single layer sensor. The sensitivity of the sensor that has four-layered thin-film (sensor B) is higher than that of the sensor that has two-layered thin-film (sensor $A$ ).

However, the sensor that has four-layered thinfilm broke easily during tests. It is necesary to overcome this disadvantage by improving the material itself or the fabrication process. 


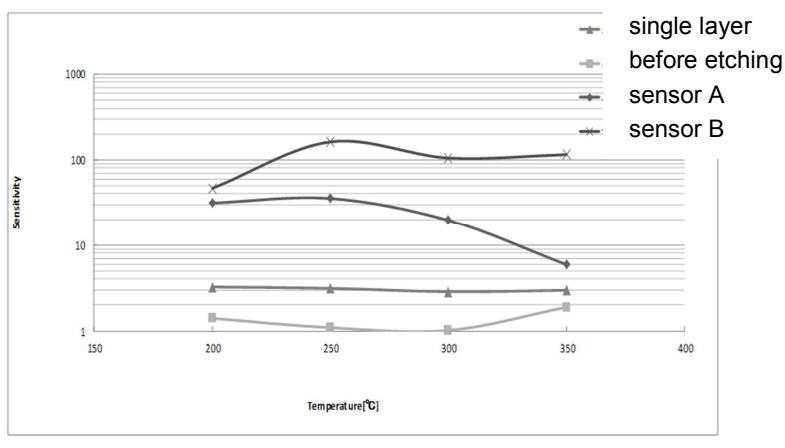

Fig.6 Temperature dependence of the sensitivity to hydrogen for 4 kinds of sensors: (1) single layer thin-film sensor, (2) two-layered thin-film sensor without an interspace (before etching), (3) two-layered thin-film sensor with an interspace (sensor A), (4) four-layered thinfilm sensor with an interspace (sensor $B$ ).

The sensitivity to various gases is shown in Fig.7 for sensor A. The sensitivity to propane gas increased after etching but the most dramatic increases in sensitivity were seen in response to hydrogen. The sensitivity to propane was $1 / 10$ of that to hydrogen. Thus the selectivity to hydrogen is satisfactory.

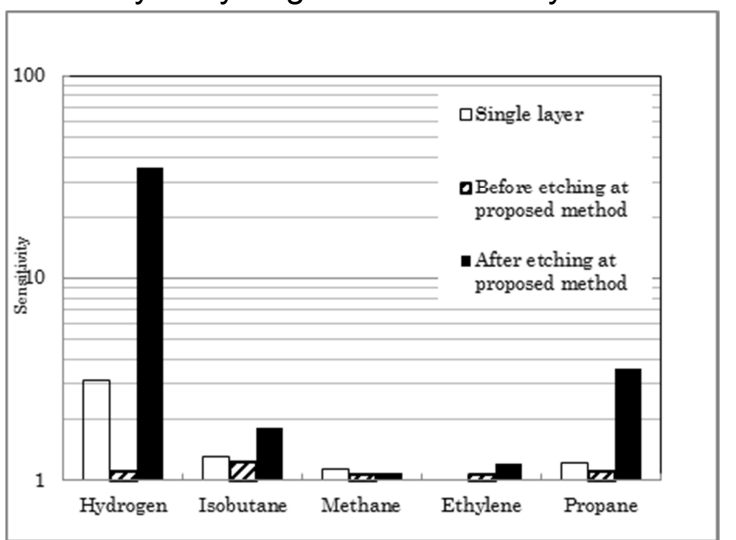

Fig. 7 Sensitivity to various gases for 3 kinds of sensors: (1) single layer thin-film sensor, (2) two-layered thin-film sensor without an interspace (before etching), (3) two-layered thin-film sensor with an interspace (sensor A). The gas concentration is $1000 \mathrm{ppm}$.

The transient response to hydrogen is shown in Fig.8. The response time and recovery time are 15 s and 20 s, respectively.

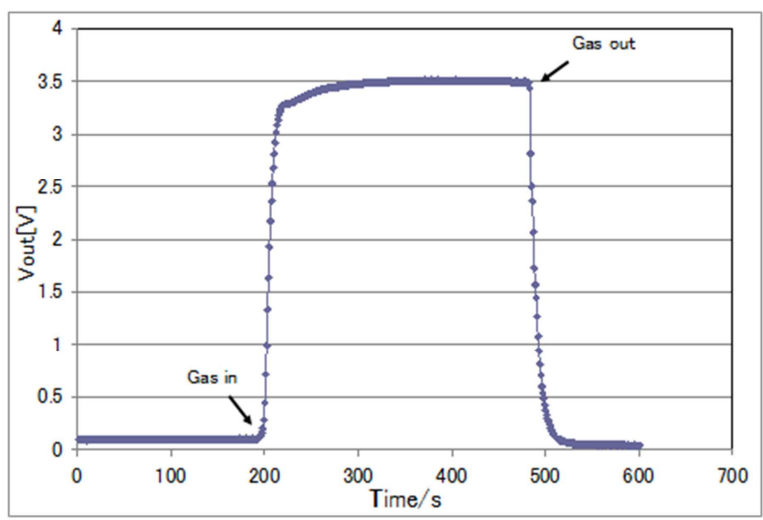

Fig. 8 Transient response to hydrogen. The gas concentration is $1000 \mathrm{ppm}$.

\section{Discussion}

An interspace in addition to the porous structure of the sensing film itself was likely to enhance the effective surface area and thus the sensitivity to hydrogen gas. The porous structure of the sensing film enables the introduction and extraction of hydrogen gas in a relatively short period of time and thus leads to a fast response of the sensor.

\section{Conclusion}

A hydrogen sensor using a multiple layered thin-film with an interspace is developed. The sensor is highly sensitive to hydrogen gas without using any noble catalysts that are expensive and may lead to degradation of the sensitivity. The material for the sensing film is $\mathrm{WO}_{3}$-based. The porous structure of the sensing film with an interspace enhances the effective surface area and thus the sensitivity to hydrogen gas beyond what can be achieved with a porous sensing film alone. The selectivity to hydrogen gas. also enhanced by the proposed structure. The response time and recovery time are 15 s and 20 s, respectively.

\section{References}

[1] T. Hubert, L. Boon-Brett, G. Black, U. Banach, Sensors and Actuators B 157, 329-352 (2011); doi: 10.1016/j.snb.2011.04.070

[2] K. Hara, N. Nishida, Sensors and Actuators B 20, 181-186 (1994) 